Buston, H. W., Jabbar, A. \& Etheridge, D. E. (1953). J.gen. Microbiol. 8, 302-306.

\title{
The Influence of Hexose Phosphates, Calcium and Jute Extract on the Formation of Perithecia by Chaetomium globosum
}

\author{
By H. W. BUSTON, A. JABBAR AND D. E. ETHERIDGE \\ Biochemical Laboratories, Department of Botany, Imperial College of \\ Science and Technology, London, S.W. 7
}

\begin{abstract}
SUMMARY : Chromatographic examination of an aqueous extract of jute showed the presence of inorganic phosphate, glucose-6-phosphate, fructose-1:6-diphosphate and calcium ion. A mixture of the last three in the amounts estimated to be present in the jute extract stimulated early and vigorous formation of perithecia of Chaetomium globosum in the same manner as does jute extract, although the amount of vegetative growth was appreciably less. No indication of the presence of a specific 'fruiting factor' was obtained.
\end{abstract}

The addition of a small amount of an aqueous extract of jute to a Czapek-Dox medium containing $0.5 \%$ glucose was shown by Buston \& Basu (1948) to affect the growth and formation of perithecia of Chaetomium globosum Kunze, in that vegetative growth became more vigorous and perithecia appeared 2 or more days earlier and in greater numbers than on the basal medium. So far as fruiting is concerned, Basu (1951) showed that a similar effect was produced by calcium within rather narrow limits of concentration (c. 10 p.p.m.), and that calcium was present in jute extract. Buston \& King (1951) noted that fruiting was accelerated by small amounts of glucose-1-phosphate or fructose1:6-diphosphate (cf. Hawker, 1948) and detected in jute extract traces of acid-labile organic phosphate, presumed to be hexose phosphates. They concluded, however, that the effect of jute extract was unlikely to be due entirely to the presence of these compounds, and pointed out that since the activity of the extract was partially destroyed by acid hydrolysis the calcium ion alone could not be responsible for the stimulation of fruiting. They did not investigate the action of calcium in combination with hexose phosphates; this is the subject of the present communication.

\section{EXPERIMENTAL}

The strain of $C$. globosum was that used previously in this laboratory, and designated by Basu no. 79. The basal medium contained (per l.): $\mathrm{NaNO}_{3}$, 2 g.; $\mathrm{KH}_{2} \mathrm{PO}_{4}, 1$ g.; KCl, 0.5 g.; $\mathrm{MgSO}_{4} .7 \mathrm{H}_{2} \mathrm{O}, 0.5$ g.; $\mathrm{FeSO}_{4} .7 \mathrm{H}_{2} \mathrm{O}, 0.01$ g.; glucose, 5 g.; agar, 20 g. All salts were Analar (British Drug Houses Ltd.) where available, or were recrystallized before use. The jute extract was prepared as described by Buston \& Basu (1948), its concentration being such that $1 \mathrm{ml}$. was equivalent to $1 \mathrm{~g}$. jute. Sterilization was done at $10 \mathrm{lb} . / \mathrm{sq} . \mathrm{in}$. for 20 min.; incubation was at $30^{\circ}$.

Using the method described by Buston \& King (1951) the combined effect 
of calcium and glucose-1-phosphate was examined. The time of appearance of perithecia was noted, and after $96 \mathrm{hr}$. an estimate of the number of perithecia formed was made by an adaptation of the method of Asthana \& Hawker (1936). These authors defined 'perithecial frequency' as the average number of perithecia/unit area of an agar culture, i.e. in a standard microscopic field. In making the counts, the cover-plate of the dish was replaced by a transparent plastic template, divided into ten equal radial sectors, which rested on top of the medium. Usually counts of three fields were made in each sector (30 fields/plate). The results (Table 1) showed that while mycelial growth and fruiting were most vigorous in the presence of jute extract, a similar acceleration of fruiting was produced by the extract and by glucose-1-phosphate, alone or in combination with calcium. Perithecial frequency was markedly increased by calcium, but was always less than that given by jute extract; also, the perithecia formed in presence of jute extract were considerably larger than those formed on the other media.

Table 1. Effect of glucose-1-phosphate and calcium on growth and sporulation of Chaetomium globosum

\begin{tabular}{|c|c|c|c|c|c|}
\hline \multirow{3}{*}{$\begin{array}{l}\text { Supplement to } \\
\text { basal medium }\end{array}$} & \multicolumn{4}{|c|}{ Time of observation (hr.) } & \multirow{3}{*}{$\begin{array}{l}\text { Perithecial } \\
\text { frequency } \\
\text { at } 96 \mathrm{hr} .\end{array}$} \\
\hline & 48 & 72 & 96 & 120 & \\
\hline & \multicolumn{4}{|c|}{ Colony diameter (mm.) } & \\
\hline Ca, 1 p.p.m. & - & $13 \cdot 6$ & $21 \cdot 1$ & $24 \cdot 5$ & - \\
\hline Ca, 10 p.p.m. & - & $11 \cdot 0$ & $19 \cdot 5$ & $23 \cdot 5 *$ & $6 \cdot 5 \dagger$ \\
\hline G-1-P, 0.05\% & $11 \cdot 6$ & $21 \cdot 3$ & $25 \cdot 6 *$ & $27 \cdot 8$ & $4 \cdot 80$ \\
\hline G-1-P $0.05 \%$ + Ca 1 p.p.m. & $13 \cdot 5$ & $21 \cdot 5$ & $24 \cdot 9 *$ & $28 \cdot 1$ & $5 \cdot 50$ \\
\hline G-1-P, $0.05 \%+$ Ca 10p.p.m. & $13 \cdot 5$ & $21 \cdot 8$ & $26 \cdot 3^{*}$ & $28 \cdot 3$ & $6 \cdot 25$ \\
\hline Jute extract & $16 \cdot 8$ & $31 \cdot 4$ & $46 \cdot 6 *$ & 65 & $7 \cdot 88$ \\
\hline
\end{tabular}

G-1-P = glucose-1-phosphate. Perithecial frequency $=$ average no. of perithecia per microscopic field (magnification $100 \times$ ).

* First appearance of perithecia.

$\dagger$ At $120 \mathrm{hr}$.

\section{Chromatographic investigation of jute extract}

The extract was examined for sugar phosphates by paper strip chromatography, using as solvent a methanol/formic acid/water mixture $(80 / 15 / 5, \mathrm{v} / \mathrm{v}$; Bandurski \& Axelrod, 1951) and spraying with the acid molybdate reagent of Hanes \& Isherwood (1949). The presence of inorganic phosphate, glucose-6phosphate and fructose-1:6-diphosphate was established; no glucose-1-phosphate was detected. Calcium was also demonstrated by the method of Pollard, McOmie \& Stevens (1951) using a spray of kojic acid and examining the dried ammonia-treated paper in ultraviolet light.

Since paper chromatography had served to separate certain components of jute extract known to be concerned with stimulating the formation of perithecia it seemed possible that further information on the relative effect of the different factors might be obtained by growing the organism directly on the paper strip after running the chromatogram, without applying the spray reagents. Accordingly, a portion of jute extract was concentrated to approxi- 
mately $1 / 5$ volume, and $0 \cdot 13 \mathrm{ml}$. portions (equivalent to the amount usually incorporated into $15 \mathrm{ml}$. of medium) were spotted on strips of filter-paper $5 \times 30 \mathrm{~cm}$. at $2 \mathrm{~cm}$. from the lower edge; ascending chromatograms were allowed to run in methanol/formic acid/water at c. $2^{\circ}$ until the solvent front had just reached the upper edge of the strip. The strips were dried in air overnight at room temperature and cut into $5 \mathrm{~cm}$. lengths, each of which was placed in a Petri dish and covered with $15 \mathrm{ml}$. of sterile basal medium; after standing for $20 \mathrm{hr}$. to allow diffusion the plates were inoculated from a young culture of $C$. globosum.

After $72 \mathrm{hr}$. perithecia were observed in three of the cultures from each strip, whilst the others showed vegetative growth only. Those sections of the strips which gave positive results corresponded to the positions occupied by the two sugar phosphates and by calcium, as shown by a control chromatogram. This result confirmed that calcium, glucose-6-phosphate and fructose1:6-diphosphate all have a stimulant effect on the formation of perithecia. It appeared very unlikely that any other constituent of jute extract was similarly effective, unless the chromatogram failed to separate it from one of these three.

\section{Attempts at quantitative measurement}

Attempts were made to utilize the formation of perithecia as a basis for estimating the amount of each stimulant in jute extract. Pieces of filter-paper $5 \mathrm{~cm}$. square were spotted with known quantities of glucose-6-phosphate, fructose-1:6-diphosphate (potassium salts) and $\mathrm{CaCl}_{2}$, placed in Petri dishes with $15 \mathrm{ml}$. sterile medium, allowed to stand for $20 \mathrm{hr}$., inoculated and incubated as usual. After $\mathbf{7 2} \mathrm{hr}$. a perithecial count was made as described above. With a strong light beneath the plate it was easily possible to make the count through the filter-paper; 30 fields/plate were counted. The results are summarized in Table 2. It appeared that for each of the three recognized stimulants there was an optimum concentration, as already observed for calcium by Basu (1951). Filter-paper itself may contain some factor which stimulates formation of perithecia (Basu, 1947). In all the present experiments the paper used (Whatman no. 1) was extracted with methanol/formic acid and dried before use, this treatment having been shown to remove the stimulant noted by Basu.

Table 2. Effects of hexose phosphates and of calcium on perithecial frequency $(\boldsymbol{P} . \boldsymbol{F}$.) in $\mathbf{7 2} \mathrm{hr}$. cultures of C. globosum

\begin{tabular}{|c|c|c|c|c|c|}
\hline \multicolumn{6}{|c|}{ Glucose-6-phosphate } \\
\hline Concn. $(\%)$ & 0.01 & 0.02 & $0 \cdot 03$ & $0 \cdot 04$ & $0 \cdot 05$ \\
\hline P.F. & $5 \cdot 2$ & $6 \cdot 6$ & $8 \cdot 8$ & $7 \cdot 6$ & $4 \cdot 7$ \\
\hline \multicolumn{6}{|c|}{ Fructose-1:6-diphosphate } \\
\hline Conen. $(\%)$ & $0 \cdot 004$ & $0 \cdot 008$ & 0.012 & $0 \cdot 016$ & 一 \\
\hline P.F. & $6 \cdot 0$ & $7 \cdot 2$ & $8 \cdot 6$ & $5 \cdot 2$ & - \\
\hline \multicolumn{6}{|c|}{ Calcium } \\
\hline Concn. (p.p.m.) & 1 & 5 & 10 & 15 & - \\
\hline P.F. & $3 \cdot 9$ & $4 \cdot 6$ & $7 \cdot 6$ & $4 \cdot 4$ & - \\
\hline
\end{tabular}


Further chromatograms were run with the jute extract, and those portions of the strip corresponding to the positions of the three recognized stimulants were tested as described above. Perithecia invariably appeared within $\mathbf{7 2} \mathbf{~ h r}$, while in control experiments their appearance was delayed by some $36-48 \mathrm{hr}$. Perithecial counts were made after $72 \mathrm{hr}$., and by comparison with curves based on the figures in Table 2, the approximate concentration of each factor was estimated. The portion containing glucose-6-phosphate gave a perithecial count of 6.4 corresponding approximately to $0.025 \%$ of the ester; that containing fructose-1:6-diphosphate gave a perithecial count of 7.7 (approximately $0.005 \%$ ester); while the calcium fraction (perithecial count $7 \cdot 1$ ) contained approximately 9.5 p.p.m. calcium.

On the basis of these results a medium was prepared to simulate the jute extract, i.e. containing 0.025 \% glucose-6-phosphate, 0.005 \% fructose-1:6diphosphate (as potassium salts) and 9.5 p.p.m. calcium (as $\mathrm{CaCl}_{2}$ ). (It is probably impossible to ensure complete absence of calcium from the materials used, and the calcium content of the medium must therefore be regarded as approximate.) Triplicate plates of this medium, triplicate plates of medium with jute extract, and plates of basal medium alone were tested in the usual manner and perithecial counts performed after $72 \mathrm{hr}$. On the jute extract medium the colonies reached a diameter of $40 \mathrm{~mm}$. in $72 \mathrm{hr}$., while those on the reconstituted medium were somewhat smaller $(35 \mathrm{~mm}$.). Perithecial frequencies were almost the same (18.6 and $\mathbf{1 8 . 3}$ respectively), but the average size of the perithecia was considerably greater in the presence of jute extract. Thus it was concluded that as to speed of formation and numbers of perithecia the defined medium was equivalent to the jute extract. In two respects, however, a difference was noted, namely that mycelial growth was significantly greater and the perithecia themselves were much larger in presence of jute extract than of the mixture of sugar phosphates + calcium (cf. p. 303). It seemed likely that the jute extract contained some nutrient or stimulant favourable to the growth of all tissues but not concerned specifically with formation of perithecia. The effect of jute extract on vegetative growth has been noted before (Buston \& Basu, 1948).

It appeared from the standard curves that the amount of each factor in the sample of jute extract used was not, except for calcium, very near its optimum concentration when acting alone. Tests were made with media in which were incorporated various combinations of the three substances in the estimated optimum quantities. In no instance did the production of perithecia equal that of a jute extract control, although the combination of all three was more effective than that of any pair. On the medium containing the two sugar phosphates (without calcium) perithecial frequency was 5.3; on that containing either phosphate + calcium, $6 \cdot 3$; on that containing all three factors, 7.3; and on the jute extract medium 12.1. (In these particular tests the perithecial frequency was unusually low even with jute extract. This was subsequently found to be due to the use of an inoculum from an older culture than usual. It was found desirable always to use an inoculum from a 7-day culture.) 


\section{DISCUSSION}

It appears that calcium ion, glucose monophosphates and fructose diphosphate may all contribute to the chemical processes which underlie the formation of perithecia, and that this (though not necessarily the total growth) is most rapid and vigorous when all three substances are present in the medium. No explanation of this observation is offered, nor of the fact that a somewhat precise balance of the three is apparently beneficial. The effect of an aqueous extract of jute fibre, which happens to provide a very effective mixture, is reproduced as regards rapidity of formation and number of perithecia by a combination of the three substances mentioned. There is no evidence of the existence in jute extract of any specific 'fruiting factor'. Glucose-1-phosphate is absent from jute extract, but appears to be about as effective as the 6-phosphate which is present; the two are probably readily interconvertible by the organism, as noted by Hawker (1948) for Melanospora destruens. Although of several natural extracts examined that from jute was most effective, it is not suggested that it provides the maximum effect possible.

We wish to thank Dr W. G. Macmillan and Dr S. N. Basu of the Indian Jute Mills Association Research Institute, Calcutta, for a generous supply of jute fibre, and Prof. W. T. J. Morgan, F.R.S., for a gift of hexose phosphates.

\section{REFERENCES}

Asthana, R. P. \& Hawker, L. E. (1936). The influence of certain fungi on the sporulation of Melanospora destruens Shear and of some other Ascomycetes. Ann. Bot., Lond. 50, 325.

BANDURski, R. S. \& Axelrod, B. (1951). The chromatographic identification of some biologically important phosphate esters. J. biol. Chem. 193, 405.

BAsu, S. N. (1947). On the sporulation of Chaetomium globosum. Thesis. University of London.

BASU, S. N. (1951). Significance of calcium in the fruiting of Chaetomium species particularly Chaetomium globosum. J. gen. Microbiol. 5, 231.

Buston, H. W. \& Basu, S. N. (1948). Some factors affecting the growth and sporulation of Chaetomium globosum and Memnoniella echinata. J. gen. Microbiol. 2, 162.

Buston, H. W. \& KInG, E. J. (1951). Further observations on the sporulation of Chaetomium globosum. J. gen. Microbiol. 5, 766.

HANES, C. S. \& Ishenwood, F. A. (1949). Separation of the phosphoric esters on the filter paper chromatogram. Nature, Lond., 164, 1107.

Hawker, L. E. (1948). Stimulation of the formation of perithecia of Melanospora destruens Shear by small quantities of certain phosphoric esters of glucose and fructose. Ann. Bot., Lond. (N.S.), 12, 77.

Pollard, F. H., McOmie, J. F. W. \& Stevens, H. M. (1951). The analysis of inorganic compounds by partition chromatography. J. chem. Soc. p. 771.

(Received 23 September 1952) 